\title{
On the nature of GRB-SGRs blazing jets
}

\author{
D. Fargion \\ Physics Dept. Rome, Univ. 1, INFN, Rome, Ple A. Moro 2, Italy \\ Received June 10; accepted July 30, 1999
}

\begin{abstract}
Gamma Ray Burst and Soft Gamma Repeaters are neither standard candle nor isotropic explosions. Our model explains them as strong blazing of a light-house, spinning and precessing gamma jet. Such jets at maximal output (as GRBs in Supernova like sources at cosmic edges) or at late lower power stages (SGRs in nearer planetary nebulae in galactic halo) may blaze the observer by extreme beaming $\left(\Omega<10^{-8}\right)$ and apparent huge luminosity.
\end{abstract}

Key words: GRB, Jet, Inverse Compton, SGR

\section{Introduction}

Gamma Ray Bursts as recent GRB 990123 and GRB 990510 emit, for isotropic explosions, energies as large as two solar masses annihilation. These energies are underestimated because of the neglected role of comparable ejected $\mathrm{MeV}$ (Comptel signal) neutrinos bursts. These extreme power cannot be explained with any standard spherically symmetric Fireball model. A too heavy black hole or Star would be unable to coexist with the shortest millisecond time structure of Gamma ray Burst. Beaming of the gamma radiation may overcome the energy puzzle. However any mild explosive beam $\left(\Omega>10^{-2}\right)$ would not solve the jet containment at the corresponding disruptive energies. Only extreme beaming $\left(\Omega<10^{-8}\right.$ ), by a slow decaying, but longlived precessing jet, it may coexist with characteristic Supernova energies, apparent GRBs output, the puzzling GRB 980425 statistics as well as their connection with older and nearer SGRs relics. Generally GRBs were understood as isotropic Fireball and SGRs are still described by isotropic galactic explosions (Magnetars). However early and late Jet models (Fargion 1994-1998; Blackmann et al. 1996) for GRBs are getting finally credit. Will be possible to accept a jet model for GRBs while keeping alive a mini fireball for SGRs? Indeed the strong SGR events (SGR 1900+14, SGR 1642-21) shared the same hard spectra of classical GRBs. One should notice the GRB-SGR similar spectra, morphology and temporal evolution within GCN/BATSE trigger 7172 GRB 981022 and 7171 GRB 981022. Nature would be extremely perverse to mimic two very comparable events at same detector, same day, by same energy spectra and comparable time structures by two totally different processes: a magnetar versus Jet GRBs. We argue here that, apart of the energetics, both of them are blazing of powerful jets (NS or $\mathrm{BH}$ ); the jets are spinning and precessing source in either binary or in accreting disk systems. The optical transient OT of GRB might be the coeval SN-like explosive birth of the jet related to its maximal intensity; the OT is absent in older relic Gamma jets, the SGRs. Their explosive memory is left around their relic nebula or plerion injected by the Gamma Jet which is running away. The late GRB OT, days after the burst, is related to the explosion intensity; it is enhanced only by a partial beaming $\left(\Omega \simeq 10^{-2}\right)$. The rarest extreme peak OT during GRB 990123 (at a million time a Supernova luminosity) may be the beamed $\left(\Omega \leq 10^{-5}\right)$ Inverse Compton optical tail, responsible of the same extreme gamma $(\mathrm{MeV})$ extreme beamed $\left(\Omega \leq 10^{-8}\right)$ signal. The huge energy bath (for a fireball model) on GRB 990123 imply a corresponding neutrino burst. As in hot universe, if entropy conservation holds, the energy density factor to be added to the photon $\gamma$ GRB 990123 budget is at least $\left(\simeq(21 / 8) \times(4 / 11)^{4 / 3}\right)$. If entropy conservation does not holds the energy needed is at least a factor [21/8] larger than the gamma one. The consequent total energy-mass needed for the two cases is respectively 3.5 and 7.2 solar masses. No fireball by NS may coexist with it. Jet could. Finally Fireballs are unable to explain the key questions related to the association GRB 980425 and SN1998bw (Galama et al. 1998): (1) Why do nearest "local" GRB 980425 in ESO 184-G82 galaxy at redshift $z_{2}=0.0083$ and the further "cosmic" GRB 971214 (Kulkarni et al. 1998) at redshift $z_{2}=3.42$ exhibit a huge average and peak intrinsic luminosity ratio? $\frac{\left\langle L_{1 \gamma}>\right.}{\left\langle L_{2 \gamma}>\right.} \cong \frac{\left\langle l_{1 \gamma}>\right.}{<l_{2 \gamma}>} \frac{z_{1}^{2}}{z_{2}^{2}} \cong 210^{5} ;\left.\frac{L_{1 \gamma}}{L_{2 \gamma}}\right|_{\text {peak }} \simeq 10^{7}$ Fluence ratios $E_{1} / E_{2}$ are also extreme $\left(\geq 410^{5}\right)$.

(2) Why is do GRB 980425 nearest event spectrum softer 
than cosmic GRB 971214 while Hubble expansion would imply the opposite by a redshift factor $\left(1+z_{1}\right) \sim 4.43$ ? (3) Why is GRB 980425 time structure slower and smoother than cosmic one, against Hubble law? (4) Why do we observe so many (even just one) nearby GRBs? Their probability to occur, with respect to a cosmic redshift $z_{1} \sim 3.42$ must be suppressed by a severe volume factor $\frac{P_{1}}{P_{2}} \cong \frac{z_{1}^{3}}{z_{2}^{3}} \simeq 710^{7}$. New GRB fireballs classes are ad hoc and fine-tuned solutions. We proposed since 1993 (Fargion 1994) that spectral and time evolution of GRB are made up of blazing beam gamma jet GJ. The GJ is born by ICS of ultrarelativistic (tens $\mathrm{GeV}$ ) electrons (pairs) on source IR, or diffused companion IR, BBR photons (Fargion, Salis 1995-1998). The target thermal photons number density may reach a few hundred to million $\mathrm{cm}^{-3}$. The thin beamed electron pair jet produced a coaxial gamma jet. It solves the GRBs energetic by the geometrical enhancement. Relativistic kinematic would imply $\theta \sim \frac{1}{\gamma_{\mathrm{e}}}$, where $\gamma_{\mathrm{e}}$ is found to reach $\gamma_{\mathrm{e}} \simeq 10^{3} \div 10^{4}$ (Fargion 1994, 1998). Unique impulsive GRB jet burst (Wang \& Wheeler 1998 ) increases the apparent luminosity by $\frac{4 \pi}{\theta^{2}} \sim 10^{7} \div 10^{9}$ but face a severe obvious probability puzzle. In particular we considered (Fargion 1998) an unique scenario where primordial GRB jets decaying in hundred and thousand years become the observable nearby SGRs. The ICS on BBR leads to GRBs spectrum (Fargion \& Salis 1995, 1996, 1998): $\frac{\mathrm{d} N_{1}}{\mathrm{~d} t_{1} \mathrm{~d} \epsilon_{1} \mathrm{~d} \Omega_{1}}$ is

$\epsilon_{1} \ln \left[\frac{1-\exp \left(\frac{-\epsilon_{1}\left(1-\beta \cos \theta_{1}\right)}{k_{\mathrm{B}} T(1-\beta)}\right)}{1-\exp \left(\frac{-\epsilon_{1}\left(1-\beta \cos \theta_{1}\right)}{k_{\mathrm{B}} T(1+\beta)}\right)}\right]\left[1+\left(\frac{\cos \theta_{1}-\beta}{1-\beta \cos \theta_{1}}\right)^{2}\right]$

scaled by a proportional factor $A_{1}$ related to the electron jet intensity. The adimensional photon number rate as a function of the observational angle $\theta_{1}$ responsible for peak luminosity becomes $\approx \theta^{-3}$. The consequent total fluence at minimal impact angle $\theta_{1 \mathrm{~m}}$ responsible for the average luminosity is $\simeq\left(\theta_{1 \mathrm{~m}}\right)^{-2}$. Assuming a beam jet intensity $I_{1}$ comparable with maximal SN luminosity, $I_{1} \simeq 10^{45} \mathrm{erg} \mathrm{s}^{-1}$, and replacing this value in adimensional $A_{1}$ in Eq. (1) we find a maximal apparent GRB power for beaming angles $10^{-3} \div 310^{-5}, P \simeq 4 \pi I_{1} \theta^{-2} \simeq$ $10^{52} \div 10^{55} \mathrm{erg} \mathrm{s}^{-1}$ within observed ones. We also assume a power law jet time decay as follows $I_{\text {jet }}=I_{1}\left(\frac{t}{t_{0}}\right)^{-\alpha} \simeq$ $10^{45}\left(\frac{t}{310^{4} \mathrm{~s}}\right)^{-1} \operatorname{erg~s}^{-1}$ where $(\alpha \simeq 1)$ able to reach, at 1000 years time scales, the present known galactic microjet (as SS433) intensities powers: $I_{\text {jet }} \simeq 10^{38} \mathrm{erg} \mathrm{s}^{-1}$. We used the model to evaluate if April precessing jet might hit us once again.

\section{GRBs 980425-980712 and SGRs-GRBs links}

Therefore the jet model answers to the puzzles (1-4): the GRB 980425 has been observed off-axis by a cone angle wider than $\frac{1}{\gamma}$ thin jet by a factor $a_{2} \sim 500$ (Fargion 1998), i.e. few degrees wide; we observed only the peripheral cone jet whose spectrum is softer and whose time structure is slower (larger impact parameter angle) and intensity strongly reduced. A simple statistics favored a repeater hit. GRB 980712 trigger 6917 was within $1.6 \sigma$ angle away from April event. Trigger 6918, repeated making the combined probability to occur quite rare $\left(\leq 10^{-3}\right)$. Because the July event has been sharper in times $(\sim 4 \mathrm{~s})$ than the April one $(\sim 20 \mathrm{~s})$, the July impact angle was smaller: $a_{3} \simeq 100$. This value is well compatible with the expected peak-average luminosity flux evolution: $\frac{L_{04 \gamma}}{L_{07 \gamma}} \simeq \frac{I_{2} \theta_{2}^{-3}}{I_{3} \theta_{3}^{-3}} \simeq$ $\left(\frac{t_{3}}{t_{2}}\right)^{-\alpha}\left(\frac{a_{2}}{a_{3}}\right)^{3} \leq 3.5$ where $t_{3} \sim 78$ day while $t_{2} \sim 210^{5} \mathrm{~s}$ The predicted fluence was also comparable with the observed ones $\frac{N_{04}}{N_{07}} \simeq \frac{\left\langle L_{04 \gamma}\right\rangle}{\left\langle L_{07 \gamma}\right\rangle} \frac{\Delta \tau_{04}}{\Delta \tau_{07}} \simeq\left(\frac{t_{3}}{t_{2}}\right)^{-\alpha}\left(\frac{a_{2}}{a_{3}}\right)^{2} \frac{\Delta \tau_{04}}{\Delta \tau_{07}} \geq$ 3. Last year 1998 SGR $1900+14$ and SGR 1627-41 events did exhibit at peak intensities spectra comparable with classical hard GRBs. We proposed their nature as the late stages of jets fueled by a disk or a companion (WD, NS) star. Their binary angular velocity $\omega_{\mathrm{b}}$ reflects the beam evolution $\theta_{1}(t)=\sqrt{\theta_{1 \mathrm{~m}}^{2}+\left(\omega_{\mathrm{b}} t\right)^{2}}$ or more generally a multiprecessing angle $\theta_{1}(t)$ (Fargion \& Salis 1996) which keeps memory of the pulsar jet spin $\left(\omega_{\mathrm{psr}}\right)$, precession by the binary $\omega_{\mathrm{b}}$ and additional nutation due to inertial momentum anisotropies or beam-accretion disk torques $\left(\omega_{\mathrm{N}}\right)$. The complicated spinning and precessing jet blazing is responsible for the wide morphology of GRBs and SGRs as well as their internal periodicity. The different geometrical observational angle might compensate the April 1998 low peak gamma luminosity $\left(10^{-7}\right)$ by a larger impact angle which compensates, at the same time, its statistical rarity $\left(\sim 10^{-7}\right)$, its puzzling softer nature and longer timescales. Such precessing jets may explain (Fargion \& Salis 1995) the external twin rings around SN1987A. We predicted its relic jet to be found in the South-East due to off-axis beaming acceleration. Jets may propel and inflate plerions as the observed ones near SRG 1647-21 and SRG 1806-20. Optical nebula NGC 6543 ("Cat Eye") and its thin jets fingers as Eta Carina ones the double cones sections in Egg nebula CRL 2688 are the most detailed and spectacular lateral view of such jets. Their blazing in-axis would appear as SGRs or, at maximal power at their SN birth, as GRBs.

\section{References}

Kulkarni S.R., et al., 1998, Nat 393, 35-39

Galama T.J., et al., 1998, astro-ph/9806175

Wang L., Wheeler J.C., 1998, astro-ph/9806212

Fargion D., 1994, R. Bernabei, WorldScientific, p. 88-97

Fargion D., Salis A., 1996, 3rd GRB: AIP. Conf. 384, 754-758

Fargion D., Salis A., 1996, 3rd GRB: AIP. Conf. 384, 749-753

Fargion D., 1998a, The Astronomers Telegram. Atel \# 31

Fargion D., 1998b, astro-ph/9808005

Fargion D., Salis A., 1995, Nuclear Phys. B (Suppl.) 43, 269273

Blackman E.G., Yi I., Field G.B., 1996, ApJ 479, L79-L82

Fargion D., Salis A., 1995, NATO ASI, 461, 397-408

Fargion D., Salis A., 1998, Physics-Uspekhi 41(8), 823-829 